\title{
Influence of the tryptophan-indole-IFN $\gamma$ axis on human genital Chlamydia trachomatis infection: role of vaginal co-infections
}

\author{
Ashok Aiyar ${ }^{*}$, Alison J. Quayle ${ }^{1}$, Lyndsey R. Buckner ${ }^{1}$, Shardulendra P. Sherchand ${ }^{1}$, \\ Theresa L. Chang, ${ }^{2}$, Arnold H. Zea ${ }^{1}$, David H. Martin ${ }^{3}$ and Robert J. Belland ${ }^{4}$ \\ ' Department of Microbiology, Immunology, and Parasitology, Louisiana State University Health Sciences Center, New Orleans, LA, USA \\ ${ }^{2}$ Department of Microbiology and Molecular Genetics, Public Health Research Institute Center, New Jersey Medical School-Rutgers, The State University of \\ New Jersey, Newark, NJ, USA \\ ${ }^{3}$ Section of Infectious Diseases, Department of Medicine, Louisiana State University Health Sciences Center, New Orleans, LA, USA \\ ${ }^{4}$ Department of Microbiology, Immunology, and Biochemistry, University of Tennessee Health Sciences Center, Memphis, TN, USA
}

\section{Edited by:}

Robert V. Schoborg, East Tennessee

State University, USA

Reviewed by:

Scott Grieshaber, University of

Florida, USA

Ted Hackstadt, Rocky Mountain Laboratories/NIAID/NIH, USA

lan Clarke, University of

Southampton, UK

*Correspondence:

Ashok Aiyar, Department of

Microbiology, Immunology, and

Parasitology, Louisiana State

University Health Sciences Center,

Medical Education Building, 1901

Perdido Street, New Orleans, LA

70112, USA

e-mail: aaiyar@Isuhsc.edu
The natural history of genital Chlamydia trachomatis infections can vary widely; infections can spontaneously resolve but can also last from months to years, potentially progressing to cause significant pathology. The host and bacterial factors underlying this wide variation are not completely understood, but emphasize the bacterium's capacity to evade/adapt to the genital immune response, and/or exploit local environmental conditions to survive this immune response. IFN $\gamma$ is considered to be a primary host protective cytokine against endocervical $C$. trachomatis infections. IFN $\gamma$ acts by inducing the host enzyme indoleamine 2,3-dioxgenase, which catabolizes tryptophan, thereby depriving the bacterium of this essential amino acid. In vitro studies have revealed that tryptophan deprivation causes Chlamydia to enter a viable but non-infectious growth pattern that is termed a persistent growth form, characterized by a unique morphology and gene expression pattern. Provision of tryptophan can reactivate the bacterium to the normal developmental cycle. There is a significant difference in the capacity of ocular and genital C. trachomatis serovars to counter tryptophan deprivation. The latter uniquely encode a functional tryptophan synthase to synthesize tryptophan via indole salvage, should indole be available in the infection microenvironment. In vitro studies have confirmed the capacity of indole to mitigate the effects of IFN $\gamma$; it has been suggested that a perturbed vaginal microbiome may provide a source of indole in vivo. Consistent with this hypothesis, the microbiome associated with bacterial vaginosis includes species that encode a tryptophanase to produce indole. In this review, we discuss the natural history of genital chlamydial infections, morphological and molecular changes imposed by IFN $\gamma$ on Chlamydia, and finally, the microenvironmental conditions associated with vaginal co-infections that can ameliorate the effects of IFN $\gamma$ on $C$. trachomatis.

Keywords: Chlamydia trachomatis, IFN $\gamma$, IDO1, tryptophan, persistence, indole, bacterial vaginosis, vaginal microbiome

\section{INTRODUCTION}

Chlamydia trachomatis is an obligate intracellular bacterium that has a unique biphasic developmental cycle. C. trachomatis serovars D through $\mathrm{K}$ are tropic for columnar epithelial cells of the urogenital tract; the endocervix is the most common site of infection in women, but organisms can ascend into the uterus and Fallopian tubes where they can cause pelvic inflammatory disease (PID) and the longer-term sequelae of tubal infertility and ectopic pregnancy. Infected, untreated women can also vertically transmit their infection to neonates, with consequences including pneumonia. Additionally, infected women have a significantly increased risk of acquiring, shedding and/or transmitting, human immunodeficiency virus-1 (HIV-1) (Wasserheit, 1992; Ghys et al., 1997; Fleming and Wasserheit, 1999; Chesson and Pinkerton, 2000; Farley et al., 2003). Despite extensive public health interventions including education, screening, and antibiotic treatment, C. trachomatis infections remain a significant global health problem, with reported U.S. cases reaching 1 million in 2006 and continuing to rise since (Centers for Disease Control, 2011). This burden of cases, together with the predominantly asymptomatic nature of the disease, have led the C. trachomatis infection to be called the "silent epidemic" (Wallis, 2001). The development of a vaccine is now considered a priori in the control of this infection.

The natural history of genital C. trachomatis infection can vary considerably. Untreated infections can be asymptomatic for substantial periods of time, progress to cause significant pathology, or spontaneously resolve without antibiotic treatment (Parks et al., 1997; Golden et al., 2000; Joyner et al., 2002; Morre et al., 2002; Molano et al., 2005; Geisler, 2010; Centers for Disease Control, 
2011). It is not understood why some C. trachomatis infections can last from months to years; clearly, the organism has the ability to survive by exploiting, adapting to, or evading, certain genital immune and environmental conditions (Brunham and Rey-Ladino, 2005). Establishing the local genital environments that enable, enhance, or deter C. trachomatis survival should allow us to: (a) identify the women at risk for extended infections; (b) define the conditions needed for the genital immune system to resolve infection naturally, and (c) aid in designing targeted vaccination and therapeutic strategies. Pertinent to this, C. trachomatis is a tryptophan auxotroph; therefore, induction of the tryptophan-catabolizing enzyme, indoleamine-2,3-dioxygenase 1 (IDO1), by the cytokine interferon gamma (IFN $\gamma$ ) restricts chlamydial growth and development in human epithelial cells (Shemer and Sarov, 1985; Byrne et al., 1986, 1989; Carlin et al., 1989; Beatty et al., 1994a; Brunham and Rey-Ladino, 2005). For this reason, IFN $\gamma$ is considered to be a major anti-chlamydial effector cytokine. In vitro, exposure of C. trachomatis-infected epithelial cells to IFN $\gamma$ can result in bacterial death, or, can cause the organism to adopt a viable but non-infectious growth mode that is termed a persistent or abnormal growth form (reviewed by Wyrick, 2010). In vitro, dependent on the culture conditions, persistent growth forms can proceed to clearance upon prolonged starvation, or they can reactivate and replicate to produce infectious elementary bodies (EBs) (Byrne et al., 1986, 1989; Beatty et al., 1993, 1994a). Pertinent to IFN $\gamma$-induced tryptophan starvation, genital, but not ocular, serovars of $C$. trachomatis have retained a functional tryptophan synthase that enables them to synthesize tryptophan via indole salvage (Fehlner-Gardiner et al., 2002; Caldwell et al., 2003). Thus, if indole is present at a sufficient concentration in the infection microenvironment, genital serovars could circumvent the bactericidal/bacteriostatic effects of IFN $\gamma$ (Beatty et al., 1993; Fehlner-Gardiner et al., 2002; Belland et al., 2003; Caldwell et al., 2003). This suggests the capacity to synthesize tryptophan in an IFN $\gamma$-rich infection microenvironment is an important virulence factor for genital C. trachomatis serovars. In vivo, the source of indole in the infection microenvironment remains unknown. However, perturbations in the vaginal flora that increase the prevalence of indole-producing bacteria during bacterial vaginosis (BV) may increase the susceptibility of women to extended infections even in the face of a robust IFN $\gamma$ response, a hypothesis originally proposed by Caldwell and coworkers in a seminal Journal of Clinical Investigation study in 2003 (Caldwell et al., 2003). However, until recent work by others and us, the nature of a "real" clinical infection, the in vivo growth characteristics of $C$. trachomatis, and the composition of the genital milieu in vivo have remained almost completely uncharacterized, precluding a test of this hypothesis. The purpose of this review is to: (a) describe the recent advances made in the characterization of the normal and perturbed vaginal microbiome that are pertinent to the indole-rescue hypothesis; (b) describe recent in vivo data to support and extend this hypothesis, including evidence that $C$. trachomatis can adopt a persistent growth mode in vivo, and that BV provides an indole-rich genital environment; and (c) explicate the mechanism by which BV and/or the vaginal Trichomonas vaginalis (TV) co-infections could modulate the effect of IFN $\gamma$ on $C$. trachomatis growth and clearance in vivo, including ramifications on clinical outcomes and choice of treatment.

\section{IFN $\gamma$-MEDIATED IMMUNITY TO GENITAL C. TRACHOMATIS INFECTION}

Several lines of evidence indicate genital C. trachomatis infection induces human immunity and that this immunity is variably protective, as reviewed recently (Batteiger et al., 2010). These include: (a) young age as a significant risk factor for infection acquisition (Arno et al., 1994); (b) reduced organism load with repeat infections (Barnes et al., 1990); (c) natural history studies documenting spontaneous resolution of infection (Parks et al., 1997; Golden et al., 2000; Joyner et al., 2002; Morre et al., 2002; Molano et al., 2005; Centers for Disease Control, 2010; Geisler, 2010); and (d) association of spontaneous resolution of infection with subsequent protection from incident disease (Geisler et al., 2013). Immunity in humans appears to develop slowly and protection from infection is generally thought to be robust only after multiple exposures and can be as short as several months (Katz et al., 1987; Molano et al., 2005), a finding corroborated in animal models (Rank and Whittum-Hudson, 2010). Further, chronic infection has been associated with pathology and the long-term sequelae of disease. Thus, significant current declines in PID are attributed to the aggressive "seek and treat" public health intervention strategies that are now in place in many developed countries (Brunham and Rekart, 2008, 2009). Paradoxically, this strategy has also been partially attributed to increasing rates of disease by blunting the development of protective immunity to this pathogen, thereby increasing the susceptibility of the population to disease (Brunham and Rekart, 2008). This has been termed the "arrested immunity" hypothesis and is corroborated by antibiotic intervention studies in mice (Su et al., 1999). Finally, in studies of sex workers who have a high risk of exposure to $C$. trachomatis via C. trachomatisinfected clients, the probability of incident C. trachomatis infection correlates inversely with duration of prostitution (Brunham et al., 1996). Finally, immune dysfunction, as indicated by HIV seropositive status, is a risk factor for incident C. trachomatis infection (ibid).

Evidence for an association of IFN $\gamma$ with genital chlamydial infection is supported by multiple experimental studies in animals (reviewed in detail in Rank and Whittum-Hudson, 2010) and observational/correlative studies in humans. Pertinently, in the murine model of genital infection using Chlamydia muridarum, a species that shares substantial genomic synteny with C. trachomatis, T-cells and IFN $\gamma$ are critical to the resolution of, and subsequent protection from, genital infection (Ramsey et al., 1988; Cain and Rank, 1995; Su et al., 1997; Johansson and Lycke, 2001). Thus, CD4, MHC class II, IFN $\gamma$ and IFN $\gamma$-receptor depletion/knockout results in chronic infection, uncontrolled $C$. muridarum bacterial burden and/or lack of protection from reinfection (Cotter et al., 1997; Perry et al., 1997; Morrison et al., 2000; Li et al., 2008; Jupelli et al., 2010; Andrew et al., 2013). Interestingly, IDO1 is poorly induced by IFN $\gamma$ in murine epithelial cells, and is not required for resolution of genital C. muridarum infection (McClarty et al., 2007). Rather, mice restrict $C$. muridarum though a cell-autonomous resistance mechanism by a 
large family of IFN $\gamma$-inducible GTPases called immunity related GTPases (Nelson et al., 2005; Miyairi et al., 2007; Coers et al., 2008; Burian et al., 2010). Consistent with the molecular mechanisms underlying IFN $\gamma$-mediated restriction in mice differing from those operant in humans, subsequent elegant molecular and cross-species studies indicate the genes that are divergent in $C$. muridarum and $C$. trachomatis strongly correlate with the ability to evade species-specific IFN $\gamma$ effector activities. Similarly comparisons of genital and ocular $C$. trachomatis serovars indicate a strong correlation with evasion of tissue-specific IFN $\gamma$ effector activities (Morrison, 2003).

In human genital infections, local cervical T-cell infiltrates and genital IFN $\gamma$ concentrations are significantly elevated during active infection, higher in women with recurrent vs. primary infection, and decreased upon resolution of infection (Figure 1), and (Arno et al., 1990; Loomis and Starnbach, 2002; Agrawal et al., 2007; Ficarra et al., 2008; Sperling et al., 2013). Local IFN $\gamma$ producing $C$. trachomatis-specific CD4 T-cells are found in the endometrium of women with a high risk of exposure to C. trachomatis (Ondondo et al., 2009). Systemically, anti-chlamydial IFN $\gamma$-producing T-cells generally peak 1-2 months after active infection in most antibiotic-treated women (Vicetti et al., 2012); however, in highly-exposed women, IFN $\gamma$-producing T-cells that recognize epitopes from $C$. trachomatis HSP60 correlate with protection against incident C. trachomatis (Cohen et al., 2005). Finally, in highly exposed HIV seropositive women, a low CD4 count is associated with low $C$. trachomatis-induced IFN $\gamma$ production (Cohen et al., 2000), and increased the risk of infection spread to the upper reproductive tract and PID (Kimani et al.,

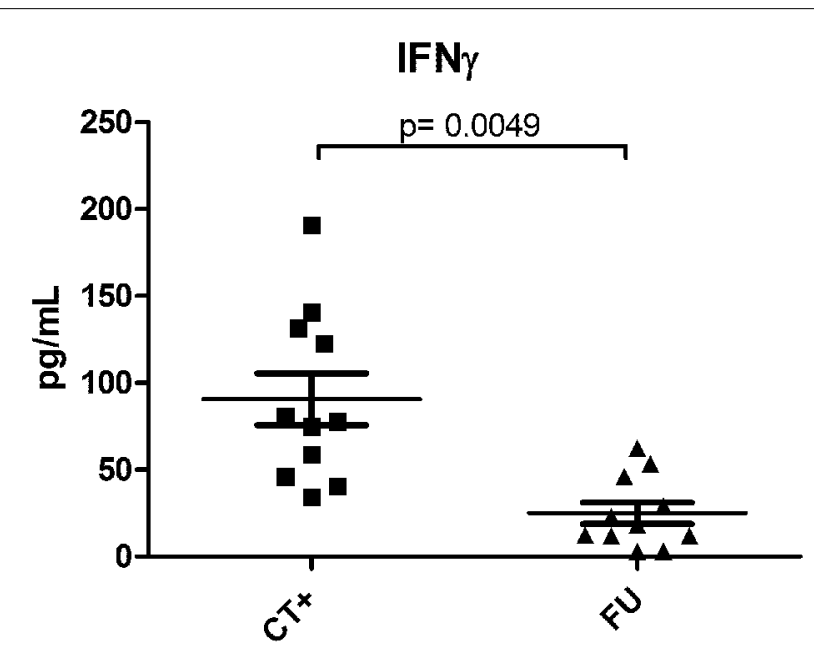

FIGURE 1 | IFN $\gamma$ levels in paired endocervical secretion samples from women during, and post-antibiotic resolution, of $\boldsymbol{C}$. trachomatis infection. Endocervical secretions, harvested as previously described (Gumbi et al., 2008), were collected from 11 women during an ongoing $\mathrm{NAAT}+C$. trachomatis infection, and again 3-6 weeks after azithromycin treatment and a confirmed NAAT- $C$. trachomatis test. Samples were quantified using multiplex cytokine analysis (Millipore), and comparisons between infected and non-infected states investigated by a

Wilcoxon-Mann-Whitney test. A $p$-value of $<0.05$ was considered significant. CT+ indicates samples from patients with an active infection and FU indicates post-antibiotic follow-up samples.
1996). While human research is challenging, we believe that new studies and tools are critical to further delineate, and mechanistically dissect, the complex relationships that exist between host immunity and bacteria in the context of the local genital environment.

\section{THE HUMAN EPITHELIAL CELL AND C. TRACHOMATIS RESPONSE TO IFN $\gamma$ AND DEPLETION OF TRYPTOPHAN}

Because C. trachomatis is a tryptophan auxotroph, IDO1mediated depletion of tryptophan curtails its growth (Taylor and Feng, 1991; Beatty et al., 1994a). C. trachomatis development is initiated when an infectious, but largely metabolically inactive, EB attaches to and enters the host cell. The internalized EB differentiates and replicates by binary fission as reticulate bodies (RBs) within a cytoplasmic membrane-bound inclusion. The completion of the developmental cycle is marked by re-differentiation of RBs into EBs that egress from the host cell. However, under conditions of limited tryptophan availability, the developmental cycle is arrested in the RB stage in a manner that results in morphologically distinct, large aberrant, viable but non-cultivable, persistent growth forms (Beatty et al., 1994a). Restoration of tryptophan availability can reactivate these persistent forms to return to normal development (Byrne et al., 1986). Similarly, provision of indole can reactivate persistent forms from genital, but not ocular, serovars, because the former synthesize a functional tryptophan synthase in response to tryptophan starvation (Caldwell et al., 2003).

\section{CHLAMYDIAL GENE EXPRESSION PATTERNS INDUCED BY IFN $\gamma$}

The induction of persistent forms with IFN $\gamma$, and their subsequent reactivation, have been studied in vitro through microarray analysis. Persistent growth, characterized by large aberrant $\mathrm{RBs}$, led to the up-regulation of genes involved in tryptophan utilization, DNA repair and recombination, phospholipid biosynthesis and translation. Up-regulation of the repressible $\operatorname{trpBA}$ operon (Belland et al., 2003) confirms the previous observations that IFN $\gamma$ treatment reduces intracellular concentrations of tryptophan. In addition, a number of early genes were upregulated, particularly the euo gene (30-fold increase), which encodes a DNA-binding protein that has been shown to bind to a late gene promoter region (i.e., omcAB Zhang et al., 1998) to repress expression. Euo has been shown to down-regulate multiple late genes (Rosario and Tan, 2012); down-regulation of $C$. trachomatis genes involved in RB to EB differentiation, proteolysis and peptide transport, and cell division were seen during persistent growth induced by IFN $\gamma$. These transcriptional analyses were consistent with the biological properties associated with aberrant RBs in that the RBs were blocked in cytokinesis, and the developmental cycle was arrested at a point preceding late gene expression. Indeed, EM analyses of persistent forms induced by IFN $\gamma$ treatment, indicates that large aberrant RBs are arrested in cytokinesis. This morphology appears to contrast quite strikingly with persistent forms induced by exposure to penicillins or the danger signal adenosine (Pettengill et al., 2009; Skilton et al., 2009), but resemble the atypical forms observed previously upon IFN $\gamma$ treatment (Beatty et al., 1993, 1994b). 
Recently, we have developed protocols that allowed the parallel assessment of both $C$. trachomatis gene transcription (specifically the euo:omcB ratio), and ultrastructure of $C$. trachomatis growth forms found in vivo in human endocervical infection (Lewis et al., 2014). The sampling protocols also permitted the quantification of indole and IFN $\gamma$ levels from the same environment (ibid). Using this multi-parameter sampling approach, we were able to visualize two strikingly different growth patterns of $C$. trachomatis growth in two patients. Specifically, one infection was characterized by morphologically normal late-stage inclusions, high viable bacterial numbers, and a low euo:omcB mRNA expression profile, together with the presence of a high concentration of indole and no IFN $\gamma$. In contrast, the second infection included morphologically aberrant forms similar in ultrastructure to in vitro persistence induced by IFN $\gamma$, a high euo:omcB mRNA expression profile, low infectious titer, and large bacterial DNA load together with a local IFN $\gamma$ response. This approach, that may be expanded to include an even more detailed analysis of C. trachomatis and host gene expression, indicate the distinct possibility of understanding how C. trachomatis grows in the genital tract, along with the environmental stresses, including IFN $\gamma$, which it encounters.

\section{TRYPTOPHAN SUPPLEMENTATION REVERSES TRANSCRIPTIONAL CHANGES INDUCED BY IFN $\gamma$}

In the in vitro model of IFN $\gamma$-induced persistence, removal of IFN $\gamma$ and supplementation with added tryptophan led to a rapid reactivation from persistent growth (Belland et al., 2003). During reactivation the expression differences rapidly returned to control levels, i.e., euo expression dropped 20 -fold in $12 \mathrm{~h}$. The transcriptional changes in the presence of IFN $\gamma$ that result in persistent growth appear to constitute a persistence stimulon. Thus, we earlier postulated that this coordinated biological response is speculated to have evolved to allow the organism to rapidly respond to immunological pressure in a manner that allows for a period of resistance followed by rapid recovery after the waning of the host response.

\section{GENITAL SEROVARS OF C. TRACHOMATIS CAN SYNTHESIZE A FUNCTIONAL TRYPTOPHAN SYNTHASE}

Shaw et al, who reported that ocular serovars encoded a truncated TrpA protein (Shaw et al., 2000), initially described differences in tryptophan synthase between ocular and genital serovars of C. trachomatis. This analysis was extended by Fehlner-Gardiner et al., who reported that genital, but not ocular, C. trachomatis serovars encode a functional, tightly regulated, tryptophan synthase $(\operatorname{trp} B A)$ permitting the bacteria to synthesize tryptophan from indole (Fehlner-Gardiner et al., 2002; Akers and Tan, 2006; Carlson et al., 2006). Along with other genes that affect pathogenesis and distinguish ocular and genital serovars of C. trachomatis (Carlson et al., 2004; Nelson et al., 2006; Taylor et al., 2010), the enzymes required for the biosynthesis of tryptophan are also encoded by genes within the plasticity zone (McClarty et al., 2007). The complement of trp genes within this region varies between chlamydial species. C. pneumoniae and C. muridarum do not encode trp genes (Xie et al., 2002a). C. psittaci lacks the $\operatorname{trp} E$ gene and is therefore incapable of synthesizing the anthranilate, an essential precursor for tryptophan biosynthesis. C. trachomatis urogenital isolates only have a subset of trp genes, encoded in the plasticity zone: $\operatorname{trp} R$, encoding a $\operatorname{trp}$ repressor; and $\operatorname{trp} A$ and $\operatorname{trp} B$, encoding homologs of the $\alpha(\operatorname{Trp} A)$ and $\beta$ (TrpB) subunits of tryptophan synthase. As reviewed recently (Raboni et al., 2009; Miles, 2013), tryptophan synthase is a tetramer consisting of two $\alpha$ subunits and two $\beta$ subunits $(\alpha 2 \beta 2)$. Functional and sequence analyses indicate that in most bacterial species that express a $\alpha 2 \beta 2$ tetramer, the enzyme is predicted to be bi-functional and catalyze the cleavage of indole glycerol3-phosphate (IGP) to indole and glyceraldehyde-3-phosphate (TrpA catalyzed $\alpha$ reaction), followed by the reaction of indole with serine to form tryptophan (TrpB catalyzed $\beta$-replacement reaction) (Xie et al., 2002b; Raboni et al., 2009; Miles, 2013). However, sequence analysis, and complementation studies conducted in E. coli, together indicate that the TrpA subunit in $C$. trachomatis appears to function structurally but not enzymatically (Fehlner-Gardiner et al., 2002; Xie et al., 2002b). As a consequence, the $\operatorname{TrpA} / \operatorname{TrpB}$ tetramer expressed by C. trachomatis during tryptophan starvation cannot use IGP as a substrate for tryptophan biosynthesis; rather, it requires indole (FehlnerGardiner et al., 2002). Therefore, indole, and not IGP, may be an important molecule in chlamydial growth by modulating the effectiveness of the IFN $\gamma$ response in bacterial clearance. Subsequent studies by Caldwell et al. verified the absolute correlation between tissue tropism and $\operatorname{trp} B A$ expression in $>200$ clinical isolates (Caldwell et al., 2003). All urogenital isolates had an intact $\operatorname{trpRBA}$ operon, while $>90$ ocular isolates invariably had frame-shift-inducing deletions within the $\operatorname{trp} A$ or $\operatorname{trp} B$ genes or had deleted the entire operon (ibid). This provided a molecular basis for the observation that only genital isolates could synthesize tryptophan from indole. While the enzymatic capacity of the chlamydial tryptophan synthase to salvage indole has been assessed only in E. coli, pioneering genetic studies reveal that a $C$. trachomatis null mutant in $\operatorname{trp} B$ has lost the capacity to escape IFN $\gamma$-mediated tryptophan depletion via indole salvage (Kari et al., 2011). Together, these data reveal a strong selective pressure for genital C. trachomatis strains to use indole salvage as a mechanism to escape IFN $\gamma$-mediated eradication by the host. Therefore, the ability to overcome tryptophan starvation may provide a mechanism through which $C$. trachomatis could cause extended and chronic infections in some women despite a robust induction of IFN $\gamma$. Critically, the availability of indole within the infection microenvironment is predicted to modulate the effect of IFN $\gamma$. Genital chlamydial infections occur in the context of the genital microbiome, of which the vaginal microbiome has been characterized in depth. The vaginal microbiome, particularly when perturbed, has been postulated to be the source of indole (Caldwell et al., 2003).

\section{AN INDOLE-POOR OCULAR MICROENVIRONMENT MAY SELECT AGAINST A FUNCTIONAL TRYPTOPHAN SYNTHASE}

Although not the focus of this review, the stark contrast in the ability of urogenital and ocular C. trachomatis isolates to express a functional tryptophan synthase is intriguing to us. Unlike the vaginal microbiome, which as described below has been characterized under a variety of conditions, the normal 
ocular microbiome is far less characterized. The two most prominent phyla in the ocular microbiome from four healthy subjects were Pseudomonas and Bradyrhizobium, neither of which produce indole (Dong et al., 2011). We note that the absence of indole in the conjunctival microenvironment is insufficient to explain why ocular chlamydial isolates have uniformly lost the capacity to express a functional tryptophan synthase, most often as a consequence of point mutations. This apparent negative selection may result from an alternative enzymatic reaction catalyzed by tryptophan synthase when indole is absent; specifically, the $\beta$-elimination reaction in which L-serine is deaminated to produce pyruvate and ammonia (Kumagai and Miles, 1971; Miles and McPhie, 1974; Xie et al., 2002b; Raboni et al., 2009). While initially described as a function of $\beta 2$ dimers, detailed studies examining the functions of the $\alpha 2 \beta 2$ tetrameric enzyme from $S$. typhimurium have revealed that it also catalyzes the $\beta$-elimination reaction necessary to produce ammonia (Ahmed et al., 1991; Raboni et al., 2005). While this catalysis is allosterically curtailed in the tetrameric enzyme by the glyceraldehyde-3-phosphate product of the $\alpha$ reaction, the $C$. trachomatis TrpA sequence, and functional analyses, indicate that it cannot bind IGP and catalyze the $\alpha$ reaction (Fehlner-Gardiner et al., 2002; Xie et al., 2002b). Further, sequence changes in the loop 6 of the C. trachomatis $\alpha$ subunit are predicted to prevent the inter-subunit interactions necessary for allosteric control (Schneider et al., 1998), consistent with the outcome of mutational analyses of the $S$. typhimurium enzyme (Yang and Miles, 1992; Brzovic et al., 1993; Kulik et al., 2002; Raboni et al., 2005). Pertinently, the activesite residues necessary for $\beta$-elimination catalysis to produce ammonia remain highly conserved in $\operatorname{TrpB}$ from $C$. trachomatis. Therefore, the $\alpha 2 \beta 2$ tetrameric enzyme from $C$. trachomatis is predicted to catalyze the generation of ammonia from serine when indole is absent. In addition to its anti-microbial effects (Rideal, 1895), ammonia is also known to induce apoptosis of epithelial cells that express the NMDA receptor (Suzuki et al., 2002; Sachs et al., 2011). In this context, the production of ammonia is currently proposed to underlie apoptosis of gastric epithelial cells induced by $H$. pylori (Seo et al., 2011). It may be of relevance that although their expression in the normal conjunctiva has not been examined, NMDA receptors are expressed in some ocular epithelial cell-lines (Oswald et al., 2012). As reviewed recently, cellular infiltrates associated with human conjunctival C. trachomatis infections include T-cells, which are capable of producing IFN $\gamma$ (Hu et al., 2013). The consequential IFN $\gamma$ induced expression of a functional tryptophan synthase in an indole-limiting environment is predicted to generate ammonia by deaminating serine, with ensuing effects that may directly select against bacterial replication or cause apoptosis of infected cells prior to completion of the normal C. trachomatis developmental cycle.

Regardless of the pressure against the expression of a functional tryptophan synthase in trachoma, the strong selective pressure on urogenital isolates to retain the capacity to synthesize tryptophan via indole salvage is striking. It is likely this pressure reflects the influence of the urogenital microbiome on C. trachomatis replication in the face of a protective immune response.

\section{THE VAGINAL MICROBIOME AND ITS EFFECTS}

It is now well appreciated that the vaginal microbiome can significantly impact the reproductive health of women, their fetuses and their newborns (Hillier et al., 1995; Zhou et al., 2010; Ma et al., 2012). Bacterial vaginosis (BV), which affects $29 \%$ of reproductive age women in the US, is characterized by the loss of Lactobacillus species and a concomitant overgrowth of diverse anaerobes (Koumans et al., 2001, 2007). BV can be diagnosed by a gram stain-based scoring system termed the Nugent score and is calculated by assessing for the presence of large Grampositive rods (chiefly Lactobacillus species), small Gram-variable rods and curved gram-variable rods; scores range from 1-10 and a Nugent of 7-10 is considered a diagnosis of BV and represents a sharp decrease in the number of Gram-positive rods with a simultaneous increase in the latter two morphotypes (Nugent et al., 1991; Delaney and Onderdonk, 2001). BV increases susceptibility to various STDs and PID (Haggerty et al., 2004) as well as the acquisition of HIV (Taha et al., 1998). Recent studies also indicate that the abnormal vaginal microbiome during BV affects the natural history of cervical human papillomavirus (HPV) and the development of cervical intraepithelial neoplasia (CIN) (Rodriguez-Cerdeira et al., 2012; Gao et al., 2013; Wheeler, 2013). Such observations indicate a dynamic relationship between the vaginal microbiome and the host; colonization by "normal" microbiomes may protect by preventing colonization by potential pathogens or by creating conditions that do not favor survival of the latter (Brotman et al., 2013). By disrupting this equilibrium, abnormal microbiomes create an environment that favors infection or colonization by various pathogens.

\section{NORMAL AND BV-ASSOCIATED VAGINAL MICROBIOMES}

Advances in high-throughput sequencing have recently permitted the development of culture-independent methods to determine the composition of vaginal microbial communities (Ravel et al., 2011). They have also permitted the evaluation of the effect of various perturbations such as antibiotics, contraceptives and sexual activity on this microbiome (Gajer et al., 2012; Brotman et al., 2013; Ravel et al., 2013). Further, the altered microbiomes present in clinically defined conditions such as BV have been characterized (Ravel et al., 2011, 2013; Datcu et al., 2013). Cross-sectional surveys using such culture-independent methods have revealed the existence of several types of vaginal communities in normal, healthy, women with distinct bacterial species compositions (Ravel et al., 2011; Ma et al., 2012; Brotman et al., 2013). Four of these communities are dominated by Lactobacillus species: Type I-L. crispatus; Type II—L. gasseri; Type III-L. iners; and Type V-L. jensenii. The fifth community type, labeled Type IV, is dominated by facultative and strict anaerobes combined with insignificant numbers of lactobacilli. The Type IV community type is most closely associated with BV, as defined by a Nugent score of 7-10. A study examining the cervical microbiome has largely recapitulated the vaginal findings (Smith et al., 2012).

\section{EFFECTS OF THE VAGINAL MICROBIOME ON C. TRACHOMATIS}

Several studies indicate the vaginal microbiome can influence $C$. trachomatis infection. For example, pregnant women with flora 
in which $\mathrm{H}_{2} \mathrm{O}_{2}$-positive Lactobacillus spp. predominate are less likely to be infected by C. trachomatis (Hillier et al., 1992). In contrast, studies using an American cohort, aged 15-30, indicated a strong correlation between BV, as defined by Nugent scores ranging from 7-10, and Chlamydia infection (odds ratio 3.4) (Wiesenfeld et al., 2003). These results were recapitulated in studies examining a Japanese cohort of similarly aged women. The latter studies found an association between BV (NS 7-10) and Chlamydia infection with an odds ratio of 3.5 (Yoshimura et al., 2009).

Differences between the normal and BV microbiome could influence the normal development of Chlamydia, and the effect of IFN $\gamma$ on normal development in multiple ways. First, we know that some members of the BV microbiome can express a functional tryptophanase to produce indole from tryptophan (Sasaki-Imamura et al., 2011), thus providing genital serovars of C. trachomatis with a means to obtain tryptophan via indole salvage. We also know from our recent studies that indole is present in the vaginal secretions of patients with BV (Lewis et al., 2014). Therefore, BV microbiome-produced indole could ameliorate the effect of IFN $\gamma$-induced tryptophan depletion on Chlamydia development. Indeed, tryptophan synthesis through indole salvage is likely to be desirable for C. trachomatis for several reasons. First, under hypoxic conditions, IDO1 can catabolize tryptophan but not indole. Second, IDO1-mediated catabolism depletes tryptophan levels within the chlamydial inclusion by decreasing extracellular and cytoplasmic tryptophan, and not by directly acting upon tryptophan within the inclusion. Third, mammalian cells lack a tryptophan synthase activity; therefore, tryptophan biosynthesis through indole salvage occurs solely within the chlamydial inclusion, providing only the bacterium with this essential amino acid with no competition from the host cell. Finally, because IDO1 catabolizes only extracellular and cytoplasmic tryptophan, it will not affect tryptophan synthesized within the inclusion.

The BV microbiome might also limit the effect of IFN $\gamma$ on $C$. trachomatis by other mechanisms. The Lactobacillus sp. that predominate in vaginal microbiome types I, II, III, and V do not synthesize indole. In addition, they create a highly acidic $\mathrm{H}_{2} \mathrm{O}_{2}$-rich environment $(\mathrm{pH}<4.2)$ that is also not conducive to chlamydial growth and development (Das et al., 2005; Haggerty et al., 2009). In contrast, anaerobes present during BV (vaginal microbiome type IV) raise the $\mathrm{pH}$ to $>4.6$ and simultaneously produce a hypoxic microenvironment. It is pertinent to note that the normal vaginal $\mathrm{pH}$ ranges from 3.8 to 4.5 . Chlamydial re-infection is favored at higher $\mathrm{pH}$, and hypoxia reduces the restrictive effect of IFN $\gamma$ on chlamydial growth in two ways: (1) Low oxygen partial pressure conditions $\left(\mathrm{pO}_{2}\right)$ limit both IFN $\gamma$-dependent signaling pathways (Roth et al., 2010); and (2) The enzymatic capacity of IDO1 to catabolize tryptophan by dioxygenation is significantly hampered by low $\mathrm{pO}_{2}$ (Herbert et al., 2011).

\section{POSSIBLE SOURCES OF INDOLE IN THE GENITAL TRACT}

Although the normal and BV microbiomes display variations between patients, there are several commonalities in their composition. The normal vagina (Nugent scores $0-3$ ) contains $\sim 10^{7}$ bacteria/10 ng of DNA recovered from vaginal swabs, in which Lactobacillus spp. predominate. During BV (Nugent score 7-10), the bacterial load is increased to $\sim 10^{9}$ bacteria/10 ng DNA, in which combinations of Prevotella spp. (>10 $8 / 10 \mathrm{ng})$, Gardnerella vaginalis $\left(10^{7} / 10 \mathrm{ng}\right)$, Atopobium vaginae $\left(10^{5} / 10 \mathrm{ng}\right)$, and/or Megasphaera spp. $\left(10^{7} / 10 \mathrm{ng}\right)$ become abundant. A large increase in the fastidious bacterium of the order Clostridiales (BVAB1$10^{8} / 10 \mathrm{ng}$ ) is also observed. Lactobacillus spp. do not produce indole, however many Prevotella spp. and strains can express a tryptophanase (tnaA) to produce indole (Sasaki-Imamura et al., 2011). Many sequenced members of the order Clostridiales also encode a tryptophanase gene. BV infections are occasionally coincident with infections by the protozoan pathogen Trichomonas vaginalis that can also express a tryptophanase to produce indole (Lloyd et al., 1991; Zubacova et al., 2011). Thus T. vaginalis coinfections may represent another mechanism by which vaginal co-infections impact the effect of IFN $\gamma$ on C. trachomatis.

These results clearly indicate that indole-producing bacteria are present within patients, with the levels of indole increasing with higher Nugent scores. Several recent studies examining the BV microbiome indicate that the increased representation of Prevotella spp. is the strongest correlate of BV (NS 7-10) (Datcu et al., 2013, 2014). Consistent with these, a metagenomic study examining mRNA expressed by the BV microbiome indicated that Prevotella spp. mRNA accounted for approximately $30 \%$ of all the mRNA expressed by the BV microbiome (Twin et al., 2013). The high representation of Prevotella spp. mRNA, coupled with the observation that some oral strains of Prevotella can express a tryptophanase, led us to test whether genital isolates of Prevotella from BV patients could also synthesize indole. For this, residual speculum fluid from three BV patients was used to isolate anaerobes using laked blood agar kanamycin/vancomycin media. Kanamycin/vancomycin-resistant constitutive anaerobes were isolated from all three samples and tested for indole production. Multiple isolates from two patients produced indole robustly, while only a single isolate from the third patient did. Partial 16S rRNA sequences (GenBank accession numbers KJ435311-KJ435324) confirmed all but one of the isolates (indole producing and non-producing) to be Prevotella spp. These results indicate that indole-producing bacteria can be found in the microbiome from the female genital tract. Further, there are variations between patients, such that even within the same genus (i.e., Prevotella), genetic differences between isolates can alter indole availability in a patient-specific manner.

\section{A GENERAL ROLE FOR INDOLE AVAILABILITY IN THE GENITAL TRACT}

Genomic and functional analyses of other bacteria indicate that indole is available in the genital tract. For example, while environmental and nosocomial infection-associated isolates of Staphylococcus aureus retain the capacity to synthesize tryptophan de novo from chorismic acid, $80 \%$ of the Toxic Shock Syndrome Toxin (TSST) producing S. aureus isolates have lost this capacity, typically due to a deletion within the $\operatorname{trpD}$ gene (McGavin et al., 2012). However, these TSS-associated isolates continue to encode a functional tryptophan synthase, suggesting that indole and/or IGP is likely available within the genital microenvironment, permitting bacterial growth even during tryptophan starvation (ibid). 
Similarly, several members of the normal and BV microbiome can synthesize tryptophan de novo from chorismic acid, others, such as G. vaginalis, resemble CT, in that they can only synthesize tryptophan by indole salvage. An analysis of the Gardnerella vaginalis reference genome (GenBank accession NC_013721) indicates it encodes a TrpB subunit that is $54 \%$ identical to the TrpB subunit from Escherichia coli, with no additional domains. However, no sequenced isolate of G. vaginalis encodes a TrpA subunit, implying that akin to CT, Gardnerella can use indole, but not IGP, in the environment to synthesize tryptophan. Therefore, it is likely that during tryptophan starvation, C. trachomatis and Gardnerella both rely on indole produced by tryptophan autotrophs that also encode a tryptophanase. For this reason, the development and use of small molecule therapeutics that target tryptophanase will not only promote IFN $\gamma$-mediated $C$. trachomatis clearance, but also aid in the clearance of indoledependent BV bacteria such as $G$. vaginalis.

\section{CONCLUDING REMARKS}

In this review, we have described the mechanism by which IFN $\gamma$ could act as a protective cytokine against chlamydial infections. IFN $\gamma$ 's protective effects result from catabolism of the essential amino-acid tryptophan by the enzyme IDO1. Depletion of tryptophan can induce a viable but not cultivable persistent growth phenotype in Chlamydia that can be reactivated when the IFN $\gamma$ response wanes and/or tryptophan is made available in the environment. Consistent with this, IFN $\gamma$ exposure induces a chlamydial pattern of gene expression that causes an up-regulation of genes that can synthesize tryptophan through indole salvage and a down-regulation of genes necessary for later stages of the chlamydial normal development cycle. Genomic analyses indicate that a selective pressure to maintain tryptophan synthesis via indole salvage has been applied strictly to every genital Chlamydia serovar. In contrast, no ocular serovars can salvage indole. Given that the genes necessary for indole salvage $(\operatorname{trpBA})$ are present in a genetic plasticity zone, it is likely that indole availability in the infection microenvironment has been the selective factor to maintain the capacity to salvage indole. Consistent with this, we have found indole in vaginal secretions from patients that have BV, and have isolated indole-producing bacteria from patients that have BV. Therefore, it is likely that natural immunity against chlamydial infections driven by a protective IFN $\gamma$ response will be attenuated in patients that have BV, dependent on the bacterial representation within individual patients. For these reasons, further studies that examine the correlates between spontaneous clearance of chlamydial infections, the host cytokine response, the host microbiome, and metabolites such as indole, are essential for the successful development of a protective vaccine against Chlamydia. Further, understanding the nature and contribution of BV-associated bacteria and their indole-producing capacity and how this relates to the effectiveness of an IFN $\gamma$-mediated resolution of $C$. trachomatis infection may also guide the management of BV in C. trachomatis-infected patients in the future.

\section{ACKNOWLEDGMENTS}

This work was supported by NIH grants AI095859 (Alison J. Quayle and Ashok Aiyar), AI070693 (Robert J. Belland) and
AI081559 (Theresa L. Chang) and by the Louisiana Vaccine Center and the South Louisiana Institute for Infectious Disease Research sponsored by the Louisiana Board of Regents.

\section{REFERENCES}

Agrawal, T., Vats, V., Wallace, P. K., Salhan, S., and Mittal, A. (2007). Cervical cytokine responses in women with primary or recurrent chlamydial infection. J. Interferon Cytokine Res. 27, 221-226. doi: 10.1089/jir.2006. 0132

Ahmed, S. A., Ruvinov, S. B., Kayastha, A. M., and Miles, E. W. (1991). Mechanism of mutual activation of the tryptophan synthase alpha and beta subunits. analysis of the reaction specificity and substrate-induced inactivation of active site and tunnel mutants of the beta subunit. J. Biol. Chem. 266, 21548-21557.

Akers, J. C., and Tan, M. (2006). Molecular mechanism of tryptophandependent transcriptional regulation in Chlamydia trachomatis. J. Bacteriol. 188, 4236-4243. doi: 10.1128/JB.01660-05

Andrew, D. W., Cochrane, M., Schripsema, J. H., Ramsey, K. H., Dando, S. J., O'meara, C. P., et al. (2013). The duration of Chlamydia muridarum genital tract infection and associated chronic pathological changes are reduced in IL-17 knockout mice but protection is not increased further by immunization. PLoS ONE 8:e76664. doi: 10.1371/journal.pone.0076664

Arno, J. N., Katz, B. P., McBride, R., Carty, G. A., Batteiger, B. E., Caine, V. A., et al. (1994). Age and clinical immunity to infections with Chlamydia trachomatis. Sex. Transm. Dis. 21, 47-52. doi: 10.1097/00007435-199401000-00010

Arno, J. N., Ricker, V. A., Batteiger, B. E., Katz, B. P., Caine, V. A., and Jones, R. B. (1990). Interferon-gamma in endocervical secretions of women infected with Chlamydia trachomatis. J. Infect. Dis. 162, 1385-1389. doi: 10.1093/infdis/162.6.1385

Barnes, R. C., Katz, B. P., Rolfs, R. T., Batteiger, B., Caine, V., and Jones, R. B. (1990). Quantitative culture of endocervical Chlamydia trachomatis. J. Clin. Microbiol. 28, 774-780.

Batteiger, B. E., Xu, F., Johnson, R. E., and Rekart, M. L. (2010). Protective immunity to Chlamydia trachomatis genital infection: evidence from human studies. J. Infect. Dis. 201(Suppl. 2), S178-S189. doi: 10.1086/652400

Beatty, W. L., Belanger, T. A., Desai, A. A., Morrison, R. P., and Byrne, G. I. (1994a). Tryptophan depletion as a mechanism of gamma interferon-mediated chlamydial persistence. Infect. Immun. 62, 3705-3711.

Beatty, W. L., Byrne, G. I., and Morrison, R. P. (1993). Morphologic and antigenic characterization of interferon gamma-mediated persistent Chlamydia trachomatis infection in vitro. Proc. Natl. Acad. Sci. U.S.A. 90, 3998-4002. doi: 10.1073/pnas.90.9.3998

Beatty, W. L., Morrison, R. P., and Byrne, G. I. (1994b). Persistent chlamydiae: from cell culture to a paradigm for chlamydial pathogenesis. Microbiol. Rev. 58, 686-699.

Belland, R. J., Nelson, D. E., Virok, D., Crane, D. D., Hogan, D., Sturdevant, D., et al. (2003). Transcriptome analysis of chlamydial growth during IFNgamma-mediated persistence and reactivation. Proc. Natl. Acad. Sci. U.S.A. 100, 15971-15976. doi: 10.1073/pnas.2535394100

Brotman, R. M., Ravel, J., Bavoil, P. M., Gravitt, P. E., and Ghanem, K. G. (2013). Microbiome, sex hormones, and immune responses in the reproductive tract: challenges for vaccine development against sexually transmitted infections. Vaccine 32, 1543-1552. doi: 10.1016/j.vaccine.2013.10.010

Brunham, R. C., Kimani, J., Bwayo, J., Maitha, G., Maclean, I., Yang, C., et al. (1996). The epidemiology of Chlamydia trachomatis within a sexually transmitted diseases core group. J. Infect. Dis. 173, 950-956. doi: 10.1093/infdis/173.4.950

Brunham, R. C., and Rekart, M. L. (2008). The arrested immunity hypothesis and the epidemiology of chlamydia control. Sex. Transm. Dis. 35, 53-54. doi: 10.1097/OLQ.0b013e31815e41a3

Brunham, R. C., and Rekart, M. L. (2009). Considerations on Chlamydia trachomatis disease expression. FEMS Immunol. Med. Microbiol. 55, 162-166. doi: 10.1111/j.1574-695X.2008.00509.x

Brunham, R. C., and Rey-Ladino, J. (2005). Immunology of Chlamydia infection: implications for a Chlamydia trachomatis vaccine. Nat. Rev. Immunol. 5, 149-161. doi: 10.1038/nri1551

Brzovic, P. S., Hyde, C. C., Miles, E. W., and Dunn, M. F. (1993). Characterization of the functional role of a flexible loop in the alpha-subunit of tryptophan synthase from Salmonella typhimurium by rapid-scanning, stopped-flow 
spectroscopy and site-directed mutagenesis. Biochemistry 32, 10404-10413. doi: 10.1021/bi00090a016

Burian, K., Endresz, V., Deak, J., Kormanyos, Z., Pal, A., Nelson, D., et al. (2010). Transcriptome analysis indicates an enhanced activation of adaptive and innate immunity by chlamydia-infected murine epithelial cells treated with interferon gamma. J. Infect. Dis. 202, 1405-1414. doi: 10.1086/656526

Byrne, G. I., Carlin, J. M., Merkert, T. P., and Arter, D. L. (1989). Long-term effects of gamma interferon on chlamydia-infected host cells: microbicidal activity follows microbistasis. Infect. Immun. 57, 1318-1320.

Byrne, G. I., Lehmann, L. K., and Landry, G. J. (1986). Induction of tryptophan catabolism is the mechanism for gamma-interferon-mediated inhibition of intracellular Chlamydia psittaci replication in T24 cells. Infect. Immun. 53, 347-351.

Cain, T. K., and Rank, R. G. (1995). Local Th1-like responses are induced by intravaginal infection of mice with the mouse pneumonitis biovar of Chlamydia trachomatis. Infect. Immun. 63, 1784-1789.

Caldwell, H. D., Wood, H., Crane, D., Bailey, R., Jones, R. B., Mabey, D., et al. (2003). Polymorphisms in Chlamydia trachomatis tryptophan synthase genes differentiate between genital and ocular isolates. J. Clin. Invest. 111, 1757-1769. doi: 10.1172/JCI17993

Carlin, J. M., Borden, E. C., and Byrne, G. I. (1989). Interferon-induced indoleamine 2,3-dioxygenase activity inhibits Chlamydia psittaci replication in human macrophages. J. Interferon Res. 9, 329-337. doi: 10.1089/jir.1989.9.329

Carlson, J. H., Hughes, S., Hogan, D., Cieplak, G., Sturdevant, D. E., McClarty, G., et al. (2004). Polymorphisms in the Chlamydia trachomatis cytotoxin locus associated with ocular and genital isolates. Infect. Immun. 72, 7063-7072. doi: 10.1128/IAI.72.12.7063-7072.2004

Carlson, J. H., Wood, H., Roshick, C., Caldwell, H. D., and McClarty, G. (2006). In vivo and in vitro studies of Chlamydia trachomatis TrpR:DNA interactions. Mol. Microbiol. 59, 1678-1691. doi: 10.1111/j.1365-2958.2006.05045.x

Centers for Disease Control. (2010). STD Health Equity: Rates by Gender. Available online at: http://www.cdc.gov/std/health-disparities/gender.htm [Accessed].

Centers for Disease Control. (2011). Sexually Transmitted Disease Surveillance: Chlamydia. Available online at: http://www.cdc.gov/std/chlamydia/stats11.htm [Accessed].

Chesson, H. W., and Pinkerton, S. D. (2000). Sexually transmitted diseases and the increased risk for HIV transmission: implications for cost-effectiveness analyses of sexually transmitted disease prevention interventions. J. Acquir. Immune Defic. Syndr. 24, 48-56. doi: 10.1097/00126334-200005010-00009

Coers, J., Bernstein-Hanley, I., Grotsky, D., Parvanova, I., Howard, J. C., Taylor, G. A., et al. (2008). Chlamydia muridarum evades growth restriction by the IFNgamma-inducible host resistance factor Irgb10. J. Immunol. 180, 6237-6245. doi: 10.4049/jimmunol.180.9.6237

Cohen, C. R., Koochesfahani, K. M., Meier, A. S., Shen, C., Karunakaran, K., Ondondo, B., et al. (2005). Immunoepidemiologic profile of Chlamydia trachomatis infection: importance of heat-shock protein 60 and interferongamma. J. Infect. Dis. 192, 591-599. doi: 10.1086/432070

Cohen, C. R., Nguti, R., Bukusi, E. A., Lu, H., Shen, C., Luo, M., et al. (2000). Human immunodeficiency virus type 1-infected women exhibit reduced interferon-gamma secretion after Chlamydia trachomatis stimulation of peripheral blood lymphocytes. J. Infect. Dis. 182, 1672-1677. doi: 10.1086/317616

Cotter, T. W., Ramsey, K. H., Miranpuri, G. S., Poulsen, C. E., and Byrne, G. I. (1997). Dissemination of Chlamydia trachomatis chronic genital tract infection in gamma interferon gene knockout mice. Infect. Immun. 65, 2145-2152.

Das, S., Sabin, C., and Allan, S. (2005). Higher vaginal pH is associated with Chlamydia trachomatis infection in women: a prospective casecontrolled study. Int. J. STD AIDS 16, 290-293. doi: 10.1258/0956462053 654221

Datcu, R., Gesink, D., Mulvad, G., Montgomery-Andersen, R., Rink, E., Koch, A., et al. (2013). Vaginal microbiome in women from Greenland assessed by microscopy and quantitative PCR. BMC Infect. Dis. 13:480. doi: 10.1186/14712334-13-480

Datcu, R., Gesink, D., Mulvad, G., Montgomery-Andersen, R., Rink, E., Koch, A., et al. (2014). Bacterial vaginosis diagnosed by analysis of firstvoid-urine specimens. J. Clin. Microbiol. 52, 218-225. doi: 10.1128/JCM. 02347-13

Delaney, M. L., and Onderdonk, A. B. (2001). Nugent score related to vaginal culture in pregnant women. Obstet. Gynecol. 98, 79-84. doi: 10.1016/S00297844(01)01402-8
Dong, Q., Brulc, J. M., Iovieno, A., Bates, B., Garoutte, A., Miller, D., et al. (2011). Diversity of bacteria at healthy human conjunctiva. Invest. Ophthalmol. Vis. Sci. 52, 5408-5413. doi: 10.1167/iovs.10-6939

Farley, T. A., Cohen, D. A., Wu, S. Y., and Besch, C. L. (2003). The value of screening for sexually transmitted diseases in an HIV clinic. J. Acquir. Immune Defic. Syndr. 33, 642-648. doi: 10.1097/00126334-200308150-00014

Fehlner-Gardiner, C., Roshick, C., Carlson, J. H., Hughes, S., Belland, R. J., Caldwell, H. D., et al. (2002). Molecular basis defining human Chlamydia trachomatis tissue tropism. a possible role for tryptophan synthase. J. Biol. Chem. 277, 26893-26903. doi: 10.1074/jbc.M203937200

Ficarra, M., Ibana, J. S., Poretta, C., Ma, L., Myers, L., Taylor, S. N., et al. (2008). A distinct cellular profile is seen in the human endocervix during Chlamydia trachomatis infection. Am. J. Reprod. Immunol. 60, 415-425. doi: 10.1111/j.1600-0897.2008.00639.x

Fleming, D. T., and Wasserheit, J. N. (1999). From epidemiological synergy to public health policy and practice: the contribution of other sexually transmitted diseases to sexual transmission of HIV infection. Sex. Transm. Infect. 75, 3-17. doi: 10.1136/sti.75.1.3

Gajer, P., Brotman, R. M., Bai, G., Sakamoto, J., Schutte, U. M., Zhong, X., et al. (2012). Temporal dynamics of the human vaginal microbiota. Sci. Transl. Med. 4, 132ra52. doi: 10.1126/scitranslmed.3003605

Gao, W., Weng, J., Gao, Y., and Chen, X. (2013). Comparison of the vaginal microbiota diversity of women with and without human papillomavirus infection: a cross-sectional study. BMC Infect. Dis. 13:271. doi: 10.1186/1471-2334-13-271

Geisler, W. M. (2010). Duration of untreated, uncomplicated Chlamydia trachomatis genital infection and factors associated with chlamydia resolution: a review of human studies. J. Infect. Dis. 201(Suppl. 2), S104-S113. doi: 10.1086/652402

Geisler, W. M., Lensing, S. Y., Press, C. G., and Hook, E. W. 3rd. (2013). Spontaneous resolution of genital Chlamydia trachomatis infection in women and protection from reinfection. J. Infect. Dis. 207, 1850-1856. doi: 10.1093/infdis/jit094

Ghys, P. D., Fransen, K., Diallo, M. O., Ettiegne-Traore, V., Coulibaly, I. M., Yeboue, K. M., et al. (1997). The associations between cervicovaginal HIV shedding, sexually transmitted diseases and immunosuppression in female sex workers in Abidjan, Cote d'Ivoire. AIDS 11, F85-F93. doi: 10.1097/00002030-19971200000001

Golden, M. R., Schillinger, J. A., Markowitz, L., and St. Louis, M. E. (2000). Duration of untreated genital infections with chlamydia trachomatis: a review of the literature. Sex. Transm. Dis. 27, 329-337. doi: 10.1097/00007435200007000-00006

Gumbi, P. P., Nkwanyana, N. N., Bere, A., Burgers, W. A., Gray, C. M., Williamson, A. L., et al. (2008). Impact of mucosal inflammation on cervical human immunodeficiency virus (HIV-1)-specific CD8 T-cell responses in the female genital tract during chronic HIV infection. J. Virol. 82, 8529-8536. doi: 10.1128/JVI.00183-08

Haggerty, C. L., Hillier, S. L., Bass, D. C., Ness, R. B., Evaluation, P. I. D., and Clinical Health Study, I. (2004). Bacterial vaginosis and anaerobic bacteria are associated with endometritis. Clin. Infect. Dis. 39, 990-995. doi: 10.1086/423963

Haggerty, C. L., Totten, P. A., Ferris, M., Martin, D. H., Hoferka, S., Astete, S. G., et al. (2009). Clinical characteristics of bacterial vaginosis among women testing positive for fastidious bacteria. Sex. Transm. Infect. 85, 242-248. doi: 10.1136/sti.2008.032821

Herbert, A., Ng, H., Jessup, W., Kockx, M., Cartland, S., Thomas, S. R., et al. (2011). Hypoxia regulates the production and activity of glucose transporter1 and indoleamine 2,3-dioxygenase in monocyte-derived endothelial-like cells: possible relevance to infantile haemangioma pathogenesis. Br. J. Dermatol. 164, 308-315. doi: 10.1111/j.1365-2133.2010.10086.x

Hillier, S. L., Krohn, M. A., Cassen, E., Easterling, T. R., Rabe, L. K., and Eschenbach, D. A. (1995). The role of bacterial vaginosis and vaginal bacteria in amniotic fluid infection in women in preterm labor with intact fetal membranes. Clin. Infect. Dis. 20(Suppl. 2), S276-S278. doi: 10.1093/clinids/20.Supplement_2.S276

Hillier, S. L., Krohn, M. A., Klebanoff, S. J., and Eschenbach, D. A. (1992). The relationship of hydrogen peroxide-producing lactobacilli to bacterial vaginosis and genital microflora in pregnant women. Obstet. Gynecol. 79, 369-373. doi: 10.1097/00006250-199203000-00008

Hu, V. H., Holland, M. J., and Burton, M. J. (2013). Trachoma: protective and pathogenic ocular immune responses to Chlamydia trachomatis. PLoS Negl. Trop. Dis. 7:e2020. doi: 10.1371/journal.pntd.0002020 
Johansson, M., and Lycke, N. (2001). Immunological memory in B-cell-deficient mice conveys long-lasting protection against genital tract infection with Chlamydia trachomatis by rapid recruitment of $\mathrm{T}$ cells. Immunology 102, 199-208. doi: 10.1046/j.1365-2567.2001.01167.x

Joyner, J. L., Douglas, J. M., Jr., Foster, M., and Judson, F. N. (2002). Persistence of Chlamydia trachomatis infection detected by polymerase chain reaction in untreated patients. Sex. Transm. Dis. 29, 196-200. doi: 10.1097/00007435200204000-00002

Jupelli, M., Selby, D. M., Guentzel, M. N., Chambers, J. P., Forsthuber, T. G., Zhong, G., et al. (2010). The contribution of interleukin-12/interferon-gamma axis in protection against neonatal pulmonary Chlamydia muridarum challenge. J. Interferon Cytokine Res. 30, 407-415. doi: 10.1089/jir.2009.0083

Kari, L., Goheen, M. M., Randall, L. B., Taylor, L. D., Carlson, J. H., Whitmire, W. M., et al. (2011). Generation of targeted Chlamydia trachomatis null mutants. Proc. Natl. Acad. Sci. U.S.A. 108, 7189-7193. doi: 10.1073/pnas.1102229108

Katz, B. P., Batteiger, B. E., and Jones, R. B. (1987). Effect of prior sexually transmitted disease on the isolation of Chlamydia trachomatis. Sex. Transm. Dis. 14, 160-164. doi: 10.1097/00007435-198707000-00008

Kimani, J., Maclean, I. W., Bwayo, J. J., Macdonald, K., Oyugi, J., Maitha, G. M., et al. (1996). Risk factors for Chlamydia trachomatis pelvic inflammatory disease among sex workers in Nairobi, Kenya. J. Infect. Dis. 173, 1437-1444. doi: 10.1093/infdis/173.6.1437

Koumans, E. H., Kendrick, J. S., and Group, C. D. C. B. V. W. (2001). Preventing adverse sequelae of bacterial vaginosis: a public health program and research agenda. Sex. Transm. Dis. 28, 292-297. doi: 10.1097/00007435-20010500000011

Koumans, E. H., Sternberg, M., Bruce, C., McQuillan, G., Kendrick, J., Sutton, M., et al. (2007). The prevalence of bacterial vaginosis in the United States, 20012004; associations with symptoms, sexual behaviors, and reproductive health. Sex. Transm. Dis. 34, 864-869. doi: 10.1097/OLQ.0b013e318074e565

Kulik, V., Weyand, M., Seidel, R., Niks, D., Arac, D., Dunn, M. F., et al. (2002). On the role of alphaThr183 in the allosteric regulation and catalytic mechanism of tryptophan synthase. J. Mol. Biol. 324, 677-690. doi: 10.1016/S00222836(02)01109-9

Kumagai, H., and Miles, E. W. (1971). The B protein of Escherichia coli tryptophan synthetase. II. new -elimination and -replacement reactions. Biochem. Biophys Res. Commun. 44, 1271-1278. doi: 10.1016/S0006-291X(71) 80223-1

Lewis, M. E., Belland, R. J., Abdelrahman, Y. M., Beatty, W., Aiyar, A., Zea, A. H., et al. (2014). Morphologic and molecular evaluation of Chlamydia trachomatis growth in human endocervix reveals distinct growth patterns. Front. Microbiol. 4:71. doi: 10.3389/fcimb.2014.00071

Li, W., Murthy, A. K., Guentzel, M. N., Seshu, J., Forsthuber, T. G., Zhong, G., et al. (2008). Antigen-specific CD4+ T cells produce sufficient IFN-gamma to mediate robust protective immunity against genital Chlamydia muridarum infection. J. Immunol. 180, 3375-3382. doi: 10.4049/jimmunol.180.5.3375

Lloyd, D., Lauritsen, F. R., and Degn, H. (1991). The parasitic flagellates Trichomonas vaginalis and Tritrichomonas foetus produce indole and dimethyl disulphide: direct characterization by membrane inlet tandem mass spectrometry. J. Gen. Microbiol. 137, 1743-1747. doi: 10.1099/00221287-137-7-1743

Loomis, W. P., and Starnbach, M. N. (2002). T cell responses to Chlamydia trachomatis. Curr. Opin. Microbiol. 5, 87-91. doi: 10.1016/S1369-5274(02)00291-6

Ma, B., Forney, L. J., and Ravel, J. (2012). Vaginal microbiome: rethinking health and disease. Annu. Rev. Microbiol. 66, 371-389. doi: 10.1146/annurev-micro092611-150157

McClarty, G., Caldwell, H. D., and Nelson, D. E. (2007). Chlamydial interferon gamma immune evasion influences infection tropism. Curr. Opin. Microbiol. 10, 47-51. doi: 10.1016/j.mib.2006.12.003

McGavin, M. J., Arsic, B., and Nickerson, N. N. (2012). Evolutionary blueprint for host- and niche-adaptation in Staphylococcus aureus clonal complex CC30. Front. Cell. Infect. Microbiol. 2:48. doi: 10.3389/fcimb.2012.00048

Miles, E. W. (2013). The tryptophan synthase alpha2beta2 complex: a model for substrate channeling, allosteric communication, and pyridoxal phosphate catalysis. J. Biol. Chem. 288, 10084-10091. doi: 10.1074/jbc.X113.463331

Miles, E. W., and McPhie, P. (1974). Evidence for a rate-determining proton abstraction in the serine deaminase reaction of the beta 2 subunit of tryptophan synthetase. J. Biol. Chem. 249, 2852-2857.

Miyairi, I., Tatireddigari, V. R., Mahdi, O. S., Rose, L. A., Belland, R. J., Lu, L., et al. (2007). The p47 GTPases Iigp2 and Irgb10 regulate innate immunity and inflammation to murine Chlamydia psittaci infection. J. Immunol. 179, 1814-1824. doi: 10.4049/jimmunol.179.3.1814

Molano, M., Meijer, C. J., Weiderpass, E., Arslan, A., Posso, H., Franceschi, S., et al. (2005). The natural course of Chlamydia trachomatis infection in asymptomatic Colombian women: a 5-year follow-up study. J. Infect. Dis. 191, 907-916. doi: $10.1086 / 428287$

Morre, S. A., van den Brule, A. J., Rozendaal, L., Boeke, A. J., Voorhorst, F. J., De Blok, S., et al. (2002). The natural course of asymptomatic Chlamydia trachomatis infections: $45 \%$ clearance and no development of clinical PID after one-year follow-up. Int. J. STD AIDS 13(Suppl. 2), 12-18. doi: $10.1258 / 095646202762226092$

Morrison, R. P. (2003). New insights into a persistent problem-chlamydial infections. J. Clin. Invest. 111, 1647-1649. doi: 10.1172/JCI18770

Morrison, S. G., Su, H., Caldwell, H. D., and Morrison, R. P. (2000). Immunity to murine Chlamydia trachomatis genital tract reinfection involves $\mathrm{B}$ cells and CD4(+) T cells but not CD8(+) T cells. Infect. Immun. 68, 6979-6987. doi: 10.1128/IAI.68.12.6979-6987.2000

Nelson, D. E., Crane, D. D., Taylor, L. D., Dorward, D. W., Goheen, M. M., and Caldwell, H. D. (2006). Inhibition of chlamydiae by primary alcohols correlates with the strain-specific complement of plasticity zone phospholipase D genes. Infect. Immun. 74, 73-80. doi: 10.1128/IAI.74.1.73-80.2006

Nelson, D. E., Virok, D. P., Wood, H., Roshick, C., Johnson, R. M., Whitmire W. M., et al. (2005). Chlamydial IFN-gamma immune evasion is linked to host infection tropism. Proc. Natl. Acad. Sci. U.S.A. 102, 10658-10663. doi: 10.1073/pnas.0504198102

Nugent, R. P., Krohn, M. A., and Hillier, S. L. (1991). Reliability of diagnosing bacterial vaginosis is improved by a standardized method of gram stain interpretation. J. Clin. Microbiol. 29, 297-301.

Ondondo, B. O., Brunham, R. C., Harrison, W. G., Kinyari, T., Sheth, P. M., Mugo, N. R., et al. (2009). Frequency and magnitude of Chlamydia trachomatis elementary body- and heat shock protein 60-stimulated interferon gamma responses in peripheral blood mononuclear cells and endometrial biopsy samples from women with high exposure to infection. J. Infect. Dis. 199, 1771-1779. doi: $10.1086 / 599095$

Oswald, D. J., Lee, A., Trinidad, M., Chi, C., Ren, R., Rich, C. B., et al. (2012). Communication between corneal epithelial cells and trigeminal neurons is facilitated by purinergic (P2) and glutamatergic receptors. PLoS ONE 7:e44574. doi: 10.1371/journal.pone.0044574

Parks, K. S., Dixon, P. B., Richey, C. M., and Hook, E. W. 3rd. (1997). Spontaneous clearance of Chlamydia trachomatis infection in untreated patients. Sex. Transm. Dis. 24, 229-235. doi: 10.1097/00007435-199704000-00008

Perry, L. L., Feilzer, K., and Caldwell, H. D. (1997). Immunity to Chlamydia trachomatis is mediated by T helper 1 cells through IFN-gamma-dependent and -independent pathways. J. Immunol. 158, 3344-3352.

Pettengill, M. A., Lam, V. W., and Ojcius, D. M. (2009). The danger signal adenosine induces persistence of chlamydial infection through stimulation of $\mathrm{A} 2 \mathrm{~b}$ receptors. PLoS ONE 4:e8299. doi: 10.1371/journal.pone.0008299

Raboni, S., Bettati, S., and Mozzarelli, A. (2005). Identification of the geometric requirements for allosteric communication between the alpha- and beta-subunits of tryptophan synthase. J. Biol. Chem. 280, 13450-13456. doi: 10.1074/jbc.M414521200

Raboni, S., Bettati, S., and Mozzarelli, A. (2009). Tryptophan synthase: a mine for enzymologists. Cell. Mol. Life Sci. 66, 2391-2403. doi: 10.1007/s00018-0090028-0

Ramsey, K. H., Soderberg, L. S., and Rank, R. G. (1988). Resolution of chlamydial genital infection in B-cell-deficient mice and immunity to reinfection. Infect. Immun. 56, 1320-1325.

Rank, R. G., and Whittum-Hudson, J. A. (2010). Protective immunity to chlamydial genital infection: evidence from animal studies. J. Infect. Dis. 201(Suppl. 2), S168-S177. doi: 10.1086/652399

Ravel, J., Brotman, R. M., Gajer, P., Ma, B., Nandy, M., Fadrosh, D. W., et al. (2013). Daily temporal dynamics of vaginal microbiota before, during and after episodes of bacterial vaginosis. Microbiome 1:29. doi: 10.1186/20492618-1-29

Ravel, J., Gajer, P., Abdo, Z., Schneider, G. M., Koenig, S. S., McCulle, S. L., et al. (2011). Vaginal microbiome of reproductive-age women. Proc. Natl. Acad. Sci. U.S.A. 108(Suppl. 1), 4680-4687. doi: 10.1073/pnas.1002611107

Rideal, S. (1895). Disinfection and Disinfectants. London: Charles Griffin and Company, Ltd. 
Rodriguez-Cerdeira, C., Sanchez-Blanco, E., and Alba, A. (2012). Evaluation of association between vaginal infections and high-risk human papillomavirus types in female sex workers in spain. ISRN Obstet. Gynecol. 2012:240190. doi: $10.5402 / 2012 / 240190$

Rosario, C. J., and Tan, M. (2012). The early gene product EUO is a transcriptional repressor that selectively regulates promoters of Chlamydia late genes. Mol. Microbiol. 84, 1097-1107. doi: 10.1111/j.1365-2958.2012.08077.x

Roth, A., Konig, P., Van Zandbergen, G., Klinger, M., Hellwig-Burgel, T., Daubener, W., et al. (2010). Hypoxia abrogates antichlamydial properties of IFN-gamma in human fallopian tube cells in vitro and ex vivo. Proc. Natl. Acad. Sci. U.S.A. 107, 19502-19507. doi: 10.1073/pnas.1008178107

Sachs, G., Marcus, E. A., and Scott, D. R. (2011). The role of the NMDA receptor in Helicobacter pylori-induced gastric damage. Gastroenterology 141, 1967-1969. doi: 10.1053/j.gastro.2011.10.019

Sasaki-Imamura, T., Yoshida, Y., Suwabe, K., Yoshimura, F., and Kato, H. (2011). Molecular basis of indole production catalyzed by tryptophanase in the genus Prevotella. FEMS Microbiol. Lett. 322, 51-59. doi: 10.1111/j.15746968.2011.02329.x

Schneider, T. R., Gerhardt, E., Lee, M., Liang, P. H., Anderson, K. S., and Schlichting, I. (1998). Loop closure and intersubunit communication in tryptophan synthase. Biochemistry 37, 5394-5406. doi: 10.1021/bi9728957

Seo, J. H., Fox, J. G., Peek, R. M. Jr., and Hagen, S. J. (2011). Nmethyl D-aspartate channels link ammonia and epithelial cell death mechanisms in Helicobacter pylori Infection. Gastroenterology 141, 2064-2075. doi: 10.1053/j.gastro.2011.08.048

Shaw, A. C., Christiansen, G., Roepstorff, P., and Birkelund, S. (2000). Genetic differences in the Chlamydia trachomatis tryptophan synthase alpha-subunit can explain variations in serovar pathogenesis. Microbes Infect. 2, 581-592. doi: 10.1016/S1286-4579(00)00368-3

Shemer, Y., and Sarov, I. (1985). Inhibition of growth of Chlamydia trachomatis by human gamma interferon. Infect. Immun. 48, 592-596.

Skilton, R. J., Cutcliffen, L. T., Barlow, D., Wang, Y., Salim, O., Lambden, P. R., et al. (2009). Penicillin induced persistence in Chlamydia trachomatis: high quality time lapse video analysis of the developmental cycle. PLoS ONE 4:e7723. doi: 10.1371/journal.pone.0007723

Smith, B. C., McAndrew, T., Chen, Z., Harari, A., Barris, D. M., Viswanathan, S., et al. (2012). The cervical microbiome over 7 years and a comparison of methodologies for its characterization. PLoS ONE 7:e40425. doi: 10.1371/journal.pone.0040425

Sperling, R., Kraus, T. A., Ding, J., Veretennikova, A., Lorde-Rollins, E., Singh, T., et al. (2013). Differential profiles of immune mediators and in vitro HIV infectivity between endocervical and vaginal secretions from women with Chlamydia trachomatis infection: a pilot study. J. Reprod. Immunol. 99, 80-87. doi: 10.1016/j.jri.2013.07.003

Su, H., Feilzer, K., Caldwell, H. D., and Morrison, R. P. (1997). Chlamydia trachomatis genital tract infection of antibody-deficient gene knockout mice. Infect. Immun. 65, 1993-1999.

Su, H., Morrison, R., Messer, R., Whitmire, W., Hughes, S., and Caldwell, H. D. (1999). The effect of doxycycline treatment on the development of protective immunity in a murine model of chlamydial genital infection. J. Infect. Dis. 180, 1252-1258. doi: 10.1086/315046

Suzuki, H., Yanaka, A., Shibahara, T., Matsui, H., Nakahara, A., Tanaka, N., et al. (2002). Ammonia-induced apoptosis is accelerated at higher $\mathrm{pH}$ in gastric surface mucous cells. Am. J. Physiol. Gastrointest. Liver Physiol. 283, G986-G995. doi: 10.1152/ajpgi.00482.2001

Taha, T. E., Dallabetta, G. A., Hoover, D. R., Chiphangwi, J. D., Mtimavalye, L. A., Liomba, G. N., et al. (1998). Trends of HIV-1 and sexually transmitted diseases among pregnant and postpartum women in urban Malawi. AIDS 12, 197-203. doi: 10.1097/00002030-199802000-00010

Taylor, L. D., Nelson, D. E., Dorward, D. W., Whitmire, W. M., and Caldwell, H. D. (2010). Biological characterization of Chlamydia trachomatis plasticity zone MACPF domain family protein CT153. Infect. Immun. 78, 2691-2699. doi: 10.1128/IAI.01455-09

Taylor, M. W., and Feng, G. S. (1991). Relationship between interferon gamma, indoleamine 2,3-dioxygenase, and tryptophan catabolism. FASEB J. 5, 2516-2522.
Twin, J., Bradshaw, C. S., Garland, S. M., Fairley, C. K., Fethers, K., and Tabrizi, S. N. (2013). The potential of metatranscriptomics for identifying screening targets for bacterial vaginosis. PLoS ONE 8:e76892. doi: 10.1371/journal.pone. 0076892

Vicetti, M., Reighard, S., Chavez, J., Rabe, L., Maryak, S., Weisenfeld, H., et al. (2012). Transient detection of Chlamydial-specific Th1 memory cells in the peripheral circulation of women with history of Chlamydia trachomatis genital tract infection. Am. J. Reprod. Immunol. 68, 499-506. doi: 10.1111/aji. 12008

Wallis, C. (2001). Chlamydia: the Silent Epidemic. Time Magazine online.

Wasserheit, J. N. (1992). Epidemiological synergy. Interrelationships between human immunodeficiency virus infection and other sexually transmitted diseases. Sex. Transm. Dis. 19, 61-77. doi: 10.1097/00007435-199219020-00001

Wheeler, C. M. (2013). The natural history of cervical human papillomavirus infections and cervical cancer: gaps in knowledge and future horizons. Obstet. Gynecol. Clin. North Am. 40, 165-176. doi: 10.1016/j.ogc.2013.02.004

Wiesenfeld, H. C., Hillier, S. L., Krohn, M. A., Landers, D. V., and Sweet, R. L. (2003). Bacterial vaginosis is a strong predictor of Neisseria gonorrhoeae and Chlamydia trachomatis infection. Clin. Infect. Dis. 36, 663-668. doi: $10.1086 / 367658$

Wyrick, P. B. (2010). Chlamydia trachomatis persistence in vitro: an overview. J. Infect. Dis. 201(Suppl. 2), S88-S95. doi: 10.1086/652394

Xie, G., Bonner, C. A., and Jensen, R. A. (2002a). Dynamic diversity of the tryptophan pathway in chlamydiae: reductive evolution and a novel operon for tryptophan recapture. Genome Biol. 3:research0051. doi: 10.1186/gb-2002-3-9research0051

Xie, G., Forst, C., Bonner, C., and Jensen, R. A. (2002b). Significance of two distinct types of tryptophan synthase beta chain in Bacteria, Archaea and higher plants. Genome Biol. 3:RESEARCH0004. doi: 10.1186/gb-2001-3-1-research0004

Yang, X. J., and Miles, E. W. (1992). Threonine 183 and adjacent flexible loop residues in the tryptophan synthase alpha subunit have critical roles in modulating the enzymatic activities of the beta subunit in the alpha 2 beta 2 complex. J. Biol. Chem. 267, 7520-7528.

Yoshimura, K., Yoshimura, M., Kobayashi, T., Kubo, T., Hachisuga, T., and Kashimura, M. (2009). Can bacterial vaginosis help to find sexually transmitted diseases, especially chlamydial cervicitis? Int. J. STD AIDS 20, 108-111. doi: 10.1258/ijsa.2008.008249

Zhang, L., Douglas, A. L., and Hatch, T. P. (1998). Characterization of a Chlamydia psittaci DNA binding protein (EUO) synthesized during the early and middle phases of the developmental cycle. Infect. Immun. 66, 1167-1173.

Zhou, X., Brotman, R. M., Gajer, P., Abdo, Z., Schuette, U., Ma, S., et al. (2010). Recent advances in understanding the microbiology of the female reproductive tract and the causes of premature birth. Infect. Dis. Obstet. Gynecol. 2010:737425. doi: 10.1155/2010/737425

Zubacova, Z., Krylov, V., and Tachezy, J. (2011). Fluorescence in situ hybridization (FISH) mapping of single copy genes on Trichomonas vaginalis chromosomes. Mol. Biochem. Parasitol. 176, 135-137. doi: 10.1016/j.molbiopara.2010.12.011

Conflict of Interest Statement: The authors declare that the research was conducted in the absence of any commercial or financial relationships that could be construed as a potential conflict of interest.

Received: 14 February 2014; accepted: 15 May 2014; published online: 03 June 2014. Citation: Aiyar A, Quayle AJ, Buckner LR, Sherchand SP, Chang TL, Zea AH, Martin $D H$ and Belland RJ (2014) Influence of the tryptophan-indole-IFNy axis on human genital Chlamydia trachomatis infection: role of vaginal co-infections. Front. Cell. Infect. Microbiol. 4:72. doi: 10.3389/fcimb.2014.00072

This article was submitted to the journal Frontiers in Cellular and Infection Microbiology.

Copyright (C) 2014 Aiyar, Quayle, Buckner, Sherchand, Chang, Zea, Martin and Belland. This is an open-access article distributed under the terms of the Creative Commons Attribution License (CC BY). The use, distribution or reproduction in other forums is permitted, provided the original author(s) or licensor are credited and that the original publication in this journal is cited, in accordance with accepted academic practice. No use, distribution or reproduction is permitted which does not comply with these terms. 